\title{
ON THE PARALLEL AND PERPENDICULAR PROPAGATING MOTIONS VISIBLE IN POLAR PLUMES: AN INCUBATOR FOR (FAST) SOLAR WIND ACCELERATION?
}

\author{
Jiajia Liu ${ }^{1,2}$, Scott W. McIntosh ${ }^{2}$, Ineke De Moortel ${ }^{3}$, And Yuming Wang ${ }^{1}$ \\ ${ }^{1}$ Earth and Space Science School, University of Science and Technology of China, NO. 96, JinZhai Road, Hefei, China \\ ${ }^{2}$ High Altitude Observatory, National Center for Atmospheric Research, P.O. Box 3000, Boulder, CO 80307, USA \\ ${ }^{3}$ School of Mathematics and Statistics, University of St. Andrews, St. Andrews, Fife, KY16 9SS, UK \\ Received 2015 March 13; accepted 2015 May 25; published 2015 June 23
}

\begin{abstract}
We combine observations of the Coronal Multi-channel Polarimeter and the Atmospheric Imaging Assembly on board the Solar Dynamics Observatory to study the characteristic properties of (propagating) Alfvénic motions and quasi-periodic intensity disturbances in polar plumes. This unique combination of instruments highlights the physical richness of the processes taking place at the base of the (fast) solar wind. The (parallel) intensity perturbations with intensity enhancements around $1 \%$ have an apparent speed of $120 \mathrm{~km} \mathrm{~s}^{-1}$ (in both the 171 and $193 \AA$ passbands) and a periodicity of 15 minutes, while the (perpendicular) Alfvénic wave motions have a velocity amplitude of $0.5 \mathrm{~km} \mathrm{~s}^{-1}$, a phase speed of $830 \mathrm{~km} \mathrm{~s}^{-1}$, and a shorter period of 5 minutes on the same structures. These observations illustrate a scenario where the excited Alfvénic motions are propagating along an inhomogeneously loaded magnetic field structure such that the combination could be a potential progenitor of the magnetohydrodynamic turbulence required to accelerate the fast solar wind.
\end{abstract}

Key words: Sun: corona - waves

Supporting material: animation

\section{INTRODUCTION}

Polar plumes are one of the most striking features in polar coronal holes (e.g., Newkirk \& Harvey 1968). Indeed, their relatively long lifetime and high contrast compared to the background coronal hole made them a favorite target of the Solar and Heliospheric Observatory era (e.g., Wilhelm 2000; Banerjee et al. 2009). They are thought of as sources of dense plasma in the fast solar wind (e.g., Gabriel et al. 2003) that result from the relentless magnetoconvective forcing of the upper solar atmospheric plasma (e.g., Wang 1998).

(Quasi-)periodic upward-propagating intensity perturbations have been observed in various regions of the Sun (e.g., Lites et al. 1999; De Moortel et al. 2002; Yamauchi et al. 2003; Banerjee et al. 2009; Liu et al. 2012) and have widely been interpreted as propagating compressional (slow) MHD waves (e.g., Ofman et al. 1999; Nakariakov 2006; Banerjee et al. 2009; De Moortel 2009). Recent progress studying spectroscopic observations (using a "blue-wing asymmetry" technique) of these (quasi-periodic) perturbations in active and quiescent regions reveals a more complex nature-it appears that at least part of the observed intensity perturbations can be attributed to mass motions (e.g., De Pontieu et al. 2009; McIntosh \& De Pontieu 2009; Tian et al. 2011). Based on the similarity analysis of the periodicity, velocity, and temperature between the quasiperiodic perturbations observed in solar polar plumes and those in other regions (active regions, coronal holes, and the quiet Sun; De Pontieu et al. 2009; McIntosh \& De Pontieu 2009), McIntosh et al. (2010) identified them as upward mass flows, which could be part of the supply of hot plasma to the fast solar wind (Parker 1991). We refer the interested reader to the review by De Moortel \& Nakariakov (2012) and the forward modeling results of De Moortel et al. (2015) for an extended discussion on the difficulties of distinguishing between the propagating waves and quasiperiodic upflows model.

Alfvénic motions in the solar atmosphere (and in polar plumes) remained undetected until the last decade when their presence was revealed in high-resolution imaging of the chromosphere with Hinode/SOT (De Pontieu et al. 2007) and the unique imaging spectroscopy capability of the Coronal Multi-channel Polarimeter (CoMP; Tomczyk et al. 2007). How these ubiquitous waves relinquish their abundant energy to the heating and/or acceleration of the plasma in the closed and open magnetic regions of the outer solar atmosphere is not well established, although a considerable body of theory (focusing on the idea of turbulence) exists (e.g., Velli 1993; Cranmer \& van Ballegooijen 2005; Verdini et al. 2010). Recently, De Moortel et al. (2014) and Liu et al. (2014) presented observations from CoMP which indicated that excess high frequency power (compared to levels expected from theoretical models) was present in counter-propagating (low-frequency) Alfvénic waves near the apex of large (transequatorial) coronal loops. These authors proposed that the relentless counter-propagation of the waves could be a potential reservoir of energy in the coronal system through MHD turbulence and cascade of wave energy from low to high frequencies.

In this paper, we present polar plume observations made by CoMP and the Atmospheric Imaging Assembly (AIA; Lemen et al. 2012) on the Solar Dynamics Observatory (SDO), exploiting their sensitivity to motions transverse to and in the plane of the sky, respectively. As in Threlfall et al. (2013), high-speed and (relatively) low-speed (longitudinal) Alfvénic motions are found to co-exist on the plumes and each process has a different periodicity. These observations shed light on the richness of the physical environment at the base of the (fast) solar wind and are used to illustrate a scenario supporting the generation of Alfvénic turbulence for the fast solar wind. 

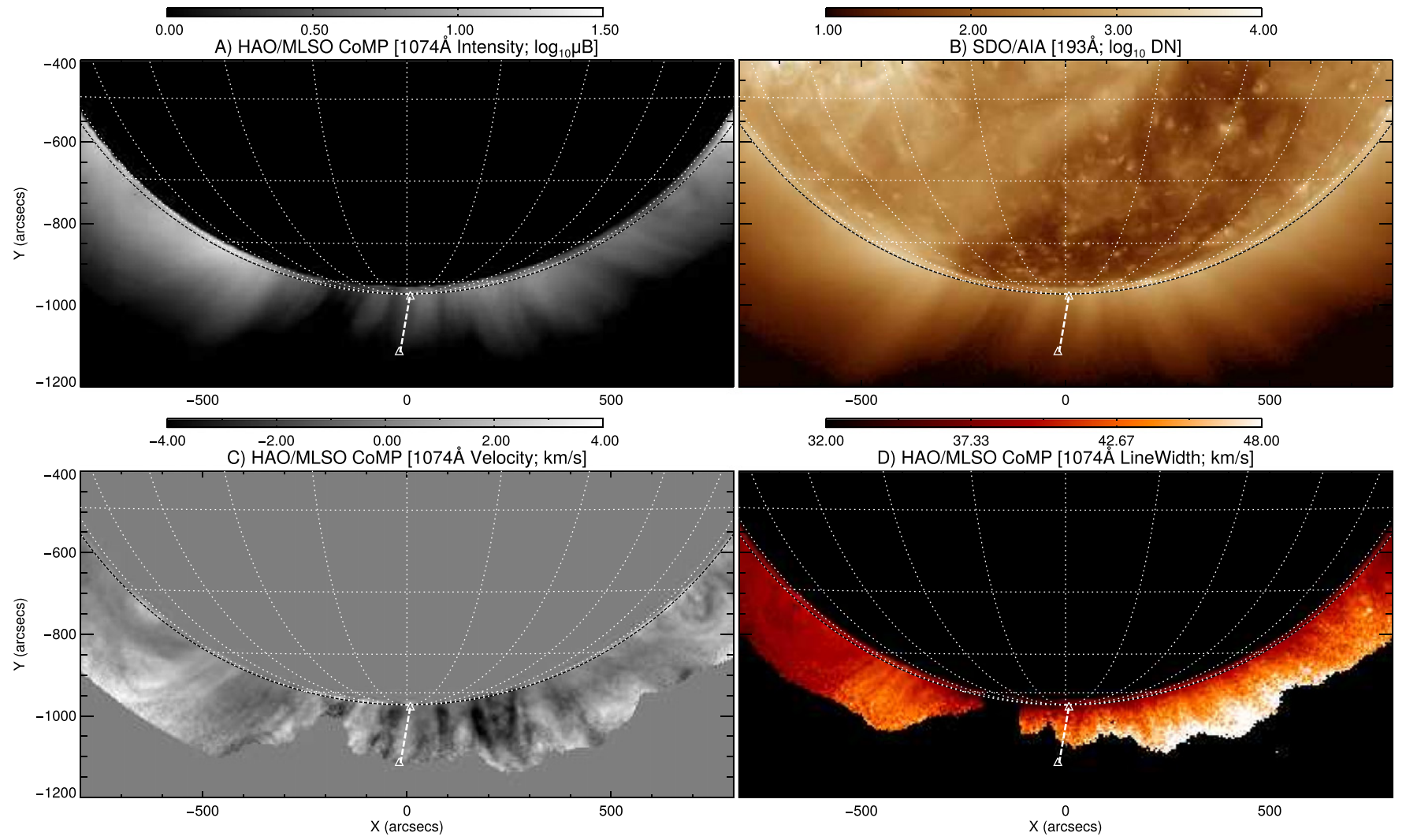

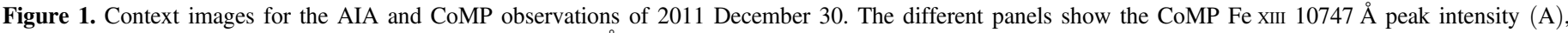

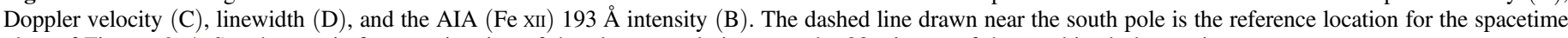
plots of Figures 2-4. See the movie for an animation of the plasma evolution over the 88 minutes of the combined observations.

(An animation of this figure is available.)

\section{OBSERVATIONS}

The CoMP (Tomczyk et al. 2008) is a combination polarimeter and narrowband tunable filter that can measure the complete polarization state in the vicinity of the 10747 and $10798 \AA$ Fe XIII coronal emission lines. The CoMP observations used in this paper were obtained in three wavelengths (10745.2, 10746.5, and 10747.8 $\mathrm{A})$ across the $10747 \AA$ Fe xIII line, with an exposure time of $250 \mathrm{~ms}$ at each position. Fitted data resulted in line peak intensity, Doppler velocity, line width, and enhanced intensity, all with a final cadence of $30 \mathrm{~s}$. The images have a full field of view (FOV) of $2.8 R_{\odot}$ and a spatial sampling of $4 " .5$.

AIA provides full disk images of the solar atmosphere, with high temporal cadence (12 s) and high spatial resolution (1".2 per pixel), extending to 1.5 solar radii. Seven narrow-band UV filters observe the Sun in a wide range of temperatures, from tens of thousands to tens of millions Kelvin, covering the atmosphere from the chromosphere to the corona. In this study, we use data from the Fe IX $171 \AA$ and Fe XII $193 \AA$ passbands, which mostly resolve material at (lower) coronal temperatures. The standard SolarSoft IDL aia_prep routine is used to read and calibrate the AIA data.

\section{ANALYSIS}

Figure 1 provides context images taken on 2011 December 30 of the (south) polar plumes we investigate in detail, with the CoMP Fe XIII $10747 \AA$ peak intensity, Doppler velocity, line width, and the AIA Fe XII $193 \AA$ intensity in panels (A), (C),
(D), and (B), respectively. A movie of the four time series together with that of the AIA Fe IX $171 \AA$ is available. The higher spatial resolution of the AIA observations is evident from a comparison with the CoMP intensity image.

The dashed line drawn near the south pole outlines the track for the spacetime plots in Figures 2-4. Figure 2(A) shows the spacetime plot of the CoMP Doppler velocities along this track using the raw data. Propagating features indicating recurring Doppler velocity perturbations with periodicity from 3 to 8 minutes can be easily seen in the spacetime diagram. The oblique blue dash-dotted lines indicate three examples of these perturbations. The lines have been shifted slightly to the right of the diagonal features in order not to obscure them. To further enhance the visibility of the Doppler velocity perturbations, a 10 time step (5 minutes) smoothed version is subtracted from the original data (Doppler velocity difference, Figure 2(B)). Alternating white and black diagonals with velocity amplitudes less than $1 \mathrm{~km} \mathrm{~s}^{-1}$ occur quasi-periodically in the spacetime plot, representing recurring upward propagating Doppler velocity perturbations along the selected track (the plume structure). The inclination of the diagonal bands represents the phase speed of these line of sight perturbations along the track, as outlined by the blue dot-dashed lines (which again have been shifted just to the right of the target features) in Figure 2(B). Some of the perturbations appear to get weaker at higher heights. This is most likely due to a combination of increased noise levels in the data, reducing the signal-to-noise but also real, physical decay of the perturbations due to mode coupling of the propagating transverse waves to azimuthal 

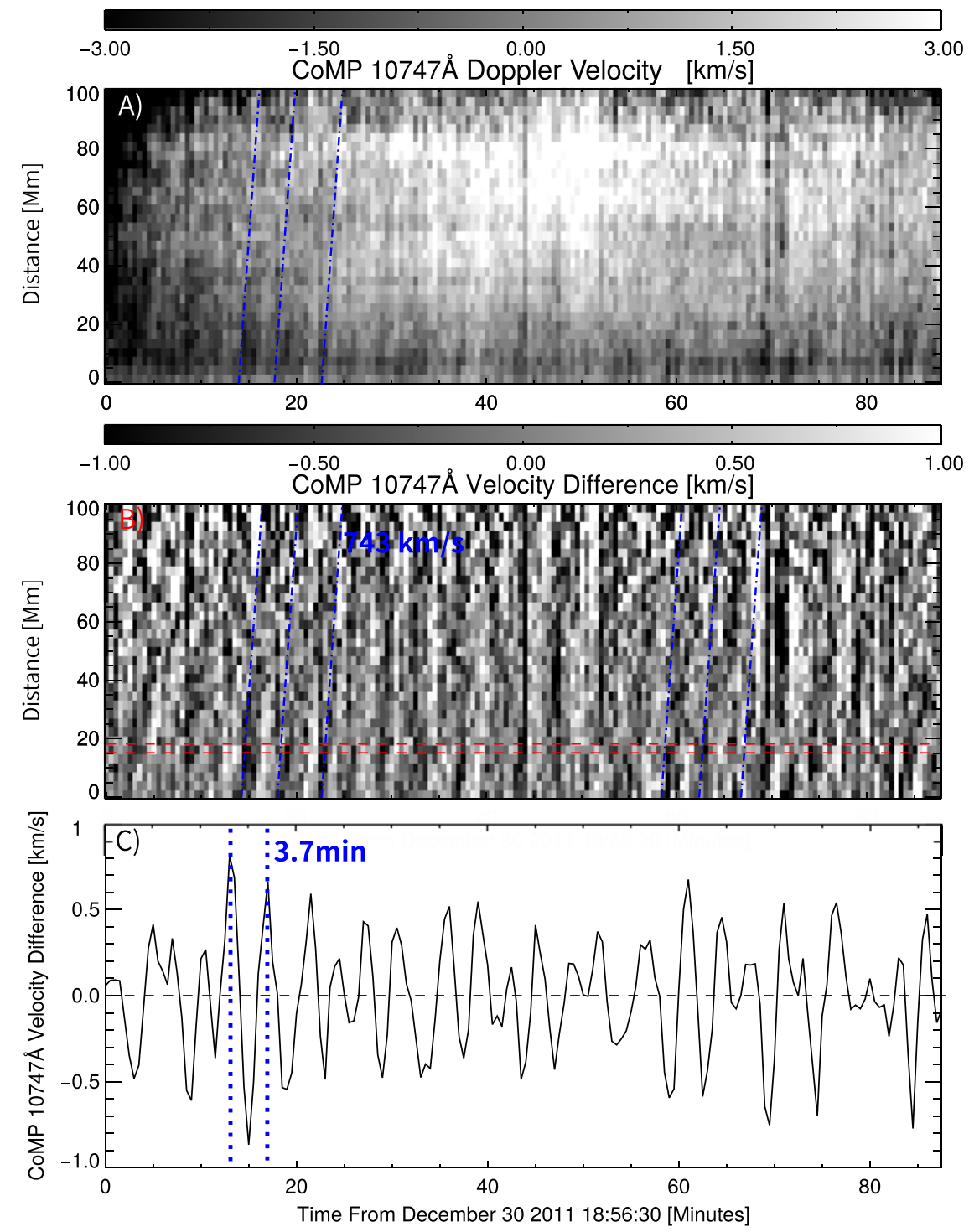

Figure 2. Panel (A): spacetime plot from the raw data of the CoMP Doppler velocity along the dashed track highlighted in Figure 1. Panel (B): corresponding spacetime plot after subtracting a 5 minute smoothed version from the original CoMP Doppler velocity data along the same track. The oblique blue dash-dotted lines in these two panels indicate several example features of propagating Doppler velocity perturbations. Panel (C): averaged velocity difference over the region confined by the two red dashed lines in panel (B). The vertical blue dotted lines indicate a typical period of the velocity perturbations.

Alfvén waves, as recently modeled by, e.g., Pascoe et al. (2010, 2013).

Figure 3 shows similar spacetime plots as the one in Figure 2(B) but after subtracting 8,14 , and 17 minute smoothed versions, respectively, from the original, raw data. These plots clearly show that the quasi-periodic perturbations are real physical features and not an artifact of the chosen smoothing interval as the recurring 3-8 minutes Doppler velocity perturbations exhibit almost the same behavior even when the smoothing interval is changed from 5 to 17 minutes.

To verify the phase speed of the propagating perturbations, we employ the same cross-correlation method as Tomczyk \& McIntosh (2009), which fits the lead/lag times versus the distance along the selected track, relative to the midpoint of the track with a straight line. The propagation speed is estimated to be about $740 \pm 107 \mathrm{~km} \mathrm{~s}^{-1}$ (with a cross-correlation factor of about 0.6), consistent with the Alfvénic wave speed obtained by Tomczyk \& McIntosh (2009) and much larger than the typical sound speed $\left(\sim 100 \mathrm{~km} \mathrm{~s}^{-1}\right.$, e.g., DeForest \& Gurman 1998) in solar polar plumes.

A spatially averaged cut across the plume in the region indicated by the two horizontal dashed red lines in Figure 2(B) is shown in panel (C). Individual events with amplitudes around $0.5 \mathrm{~km} \mathrm{~s}^{-1}$ occur quasi-periodically with a recurrence rate from 3 to 8 minutes, consistent with our visual estimate from the spacetime plots. Wavelet analysis (Torrence \& Compo 1998) on the time series reveals a dominant period of about $3.7 \pm 0.7$ minutes. As in previous studies, no corresponding intensity perturbations (with similar periodicity) are observed in the CoMP or AIA intensity observations, indicating the largely non-compressive (Alfvénic) nature of the disturbances.

Subsequently, we also perform the same analysis on the AIA observations. Figures 4(A) and (C) show the corresponding spacetime plots of the AIA 193 and $171 \AA$ intensity observations along the selected track, respectively. 

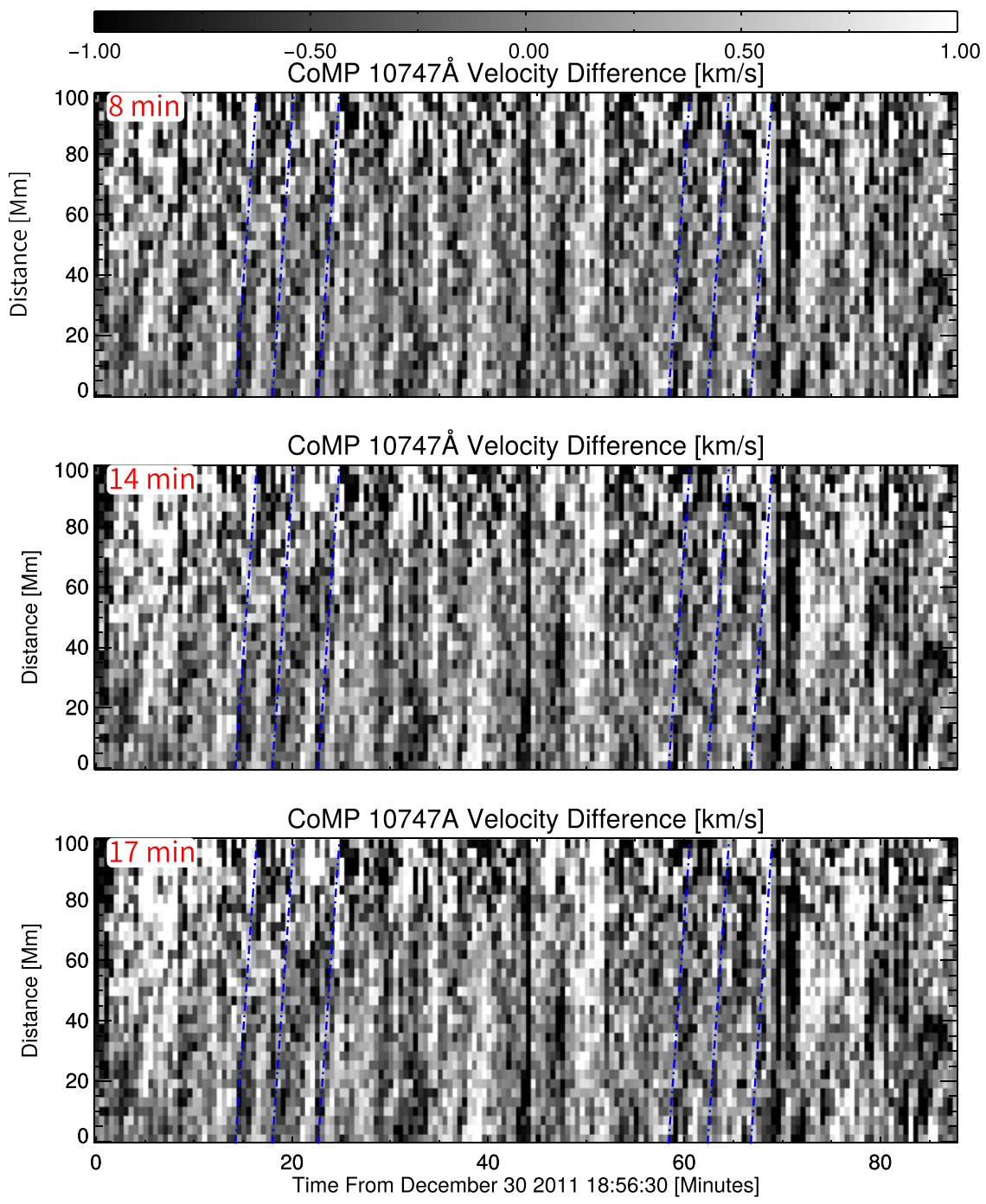

Figure 3. Spacetime plots as panel (B) in Figure 2 but after subtracting 8, 14, and 17 minute smoothed versions, respectively. The oblique blue, dash-dotted lines are exactly the same as those in Figure 2(B).

The AIA $193 \AA$ spacetime plot clearly shows recurring, propagating intensity perturbations (diagonal green and red bands), but with a much longer period than the CoMP Doppler velocity perturbations observed along the same track, suggesting that several different physical processes might be taking place along (or within) this plume structure. The upward propagating AIA intensity perturbations are of the order of $1 \%$ (compared to the background intensity, taken as a 5 minute smoothed version of the initial data) and have propagation speeds of about $130 \mathrm{~km} \mathrm{~s}^{-1}$ (see Banerjee et al. 2011 for a review of similar intensity disturbances propagating along coronal plumes). Wavelet analysis reveals a dominant period of about 12 minutes, which is indeed considerably longer than that of the Doppler perturbations. The AIA $171 \AA$ passband data reveal almost identical results.

Applying the same approach on 13 other plume structures in the south polar coronal hole yields similar results, namely the combination of fast-propagating Doppler velocity perturbations and low amplitude, slower intensity perturbations travelling along all the plumes. Table 1 shows the observed properties for all 14 plumes. Statistical analysis clearly shows the very different propagating speeds $\left(834 \pm 203 \mathrm{~km} \mathrm{~s}^{-1}\right.$ versus $\left.120 \pm 18 \mathrm{~km} \mathrm{~s}^{-1}\right)$ and periods $(4.8 \pm 0.9$ minutes versus
$15.2 \pm 5.0$ minutes). Careful inspection of the movie provided suggests the ubiquitous presence of these high-speed Doppler velocity perturbations and weaker and slower intensity perturbations along most plumes in the field of view.

\section{DISCUSSIONS}

The observational analysis presented above demonstrated the co-existence of two characteristic types of perturbations in solar polar plumes: transverse (line of sight) Doppler velocity perturbations observed by the CoMP instrument, interpreted as propagating Alfvénic waves and weak, parallel (longitudinal), quasi-periodic, propagating disturbances in the AIA intensities. The combination of these different types of perturbations, coexisting on the same (plume) structure, indicates a richness of different physical processes taking place, at or near the potential source region of the fast solar wind. A similar coexistence of different perturbations was also found by Threlfall et al. (2013) along large coronal loops.

The observed Alfvénic motions propagate with phase speeds around $830 \pm 200 \mathrm{~km} \mathrm{~s}^{-1}$, similar to Alfvénic perturbations observed before in the solar corona (e.g., Tomczyk \& McIntosh 2009; De Moortel et al. 2014). The (Doppler) perturbation 

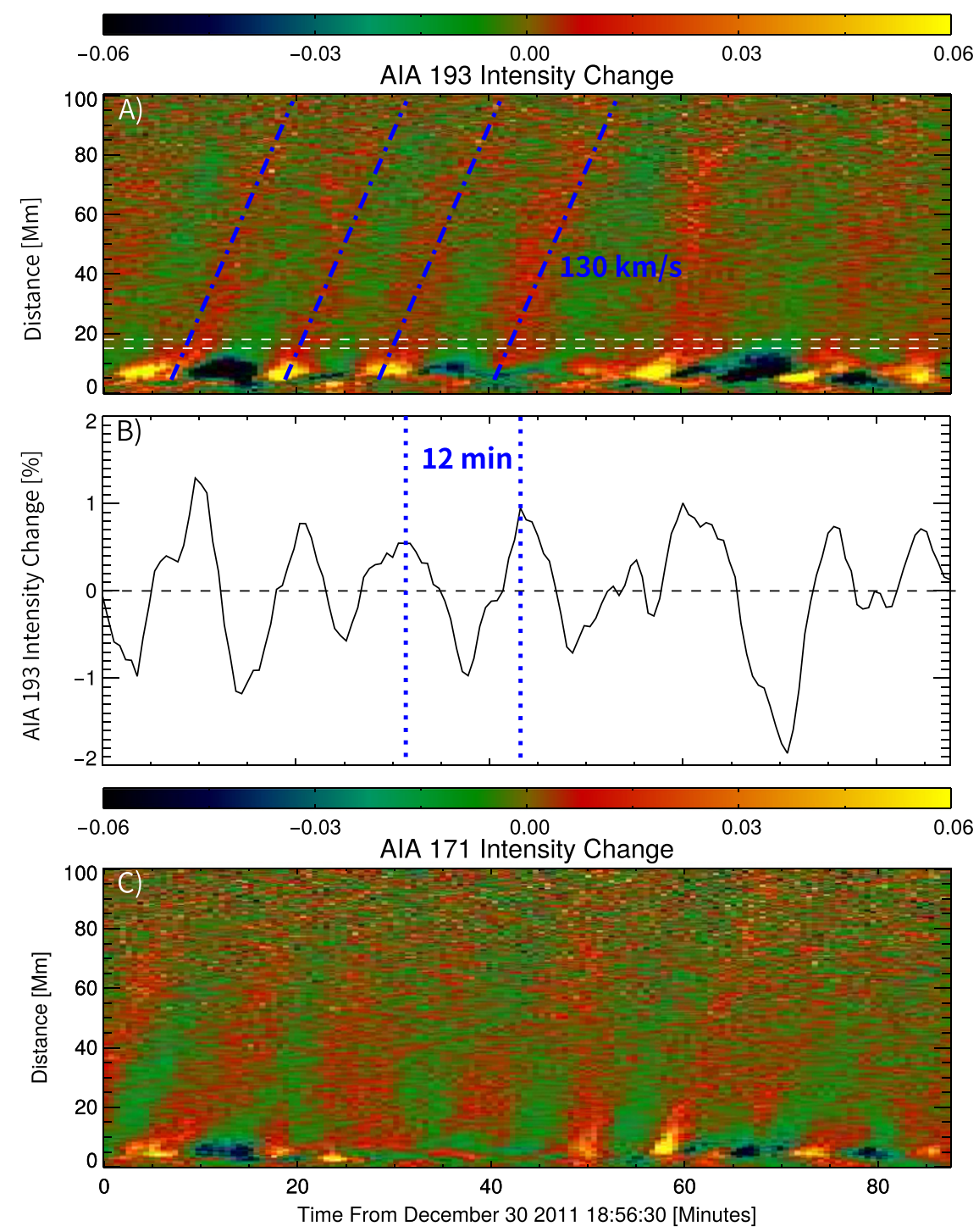

Figure 4. Panel (A): spacetime plot of the AIA $193 \AA$ Antensity changes along the dashed track highlighted in Figure 1 . The diagonal blue dash-dotted lines indicate the phase speeds of the intensity perturbations. Panel (B): averaged velocity difference over the region outlined by the two white dashed lines in panel (A). The vertical blue dotted lines indicate a typical period of the intensity perturbations. Panel (C): corresponding spacetime plot of the AIA $171 \AA$ Antensity changes along the same track.

amplitudes are of the order of $0.5 \mathrm{~km} \mathrm{~s}^{-1}$, with no obvious corresponding (observed) intensity variation. To first order, the energy flux carried by these waves can be estimated as $F_{W}=\rho\left\langle v^{2}>V_{\text {phase }}\right.$, where $\rho$ is the plasma density, $v$ the velocity amplitude, and $V_{\text {phase }}$ the phase speed (see also Goossens et al. 2013 for a discussion on energy content in Alfvénic waves). As demonstrated in McIntosh \& De Pontieu (2012) and De Moortel \& Pascoe (2012), perturbation amplitudes obtained from the Doppler velocities could substantially underestimate the true velocity amplitudes (and hence energy flux) due to the relatively low (spatial) resolution and/or the line of sight superposition effects. Using observed line-widths and comparing with Monte-Carlo simulations, McIntosh \& De Pontieu (2012) estimated that the true amplitudes of the perturbations could be on the order of $25-56 \mathrm{~km} \mathrm{~s}^{-1}$. With typical values in solar polar plumes for the electron number density of the order of $10^{8} \mathrm{~cm}^{-3}$, and the phase speed $\sim 830 \mathrm{~km} \mathrm{~s}^{-1}$, we obtain an energy flux carried by the observed Alfvénic waves of about $80-400 \mathrm{~W} \mathrm{~m}^{-2}$, sufficient to balance the estimated loss of about $\sim 100 \mathrm{~W} \mathrm{~m}^{-2}$ (Withbroe \& Noyes 1977).

Upward-propagating intensity perturbations are observed in the AIA 193 and $171 \AA$ images, co-existing on the plumes with the Alfvénic wave motions, but with much slower propagation speeds of $\sim 120 \mathrm{~km} \mathrm{~s}^{-1}$, which is on the order of the theoretically expected slow magnetoacoustic speed in the solar polar region (e.g., DeForest \& Gurman 1998). Similar lowamplitude, quasi-periodic intensity perturbations are commonly observed in the solar atmosphere and we refer the reader to, for example, De Moortel (2009) or Banerjee et al. (2011) for a review. Early observations (mostly using only imaging observations) interpreted the observed perturbations as propagating, slow magneto-acoustic waves. However, recent spectroscopic observations (e.g., De Pontieu et al. 2009; McIntosh et al. 2010) have revealed a more complex picture, indicating a mass motion component. Forward modeling of numerical simulations by De Moortel et al. (2015) highlighted the fundamental difficulty of distinguishing between these two 
Table 1

Properties of the Perturbations Observed along 14 Plumes observed on 2011 December 30

\begin{tabular}{|c|c|c|c|c|c|c|}
\hline \multirow{2}{*}{ No. } & \multicolumn{3}{|c|}{ CoMP Doppler Velocity } & \multicolumn{3}{|c|}{ AIA $193 \AA$ Intensity } \\
\hline & $v_{d}\left(\mathrm{~km} \mathrm{~s}^{-1}\right)$ & $P_{d}$ (minutes) & $v_{p d}\left(\mathrm{~km} \mathrm{~s}^{-1}\right)$ & $I_{e}(\%)$ & $P_{a}$ (minutes) & $v_{p a}\left(\mathrm{~km} \mathrm{~s}^{-1}\right)$ \\
\hline 1 & 0.42 & 4.9 & 1021.7 & 0.97 & 20.1 & 113.6 \\
\hline 4 & 0.51 & 4.3 & 1149.0 & 0.92 & 15.5 & 80.7 \\
\hline 5 & 0.50 & 5.0 & 976.9 & 0.99 & 14.1 & 114.3 \\
\hline 6 & 0.58 & 5.6 & 607.5 & 0.51 & 14.4 & 116.5 \\
\hline 9 & 0.38 & 5.4 & 932.1 & 0.70 & 20.4 & 131.6 \\
\hline 10 & 0.45 & 6.9 & 818.0 & 1.27 & 14.0 & 100.8 \\
\hline 11 & 0.40 & 5.9 & 690.5 & 0.69 & 10.7 & 130.8 \\
\hline 12 & 0.39 & 5.8 & 633.5 & 0.65 & 15.0 & 147.6 \\
\hline 13 & 0.43 & 4.6 & 906.5 & 1.40 & 16.0 & 120.2 \\
\hline 14 & 0.43 & 4.4 & 1225.0 & 0.47 & 13.6 & 149.0 \\
\hline
\end{tabular}

Notes. $v_{d}, P_{d}$, and $v_{p d}$ are the peak Doppler velocity difference, the period, and the phase speed of the perpendicular wave motions observed in the CoMP Doppler velocity images, respectively. $I_{e}, P_{a}$, and $v_{p a}$ are the peak intensity enhancement, the periodicity, and the propagation speed of the longitudinal intensity disturbances in the AIA $193 \AA$ images, respectively. Example No. 2 is the plume discussed in detail in this paper.

different interpretations (slow propagating waves and quasiperiodic upflows). However, regardless of whether these intensity perturbations are quasi-periodic upflows or slow magnetoacoustic waves, the intensity (density) enhancements might cause reflection of the fast Alfvénic waves and the interaction of the reflected wave trains could lead to the onset of an Alfvénic turbulent cascade. Such a turbulent cascade would enhance the dissipation of Alfvénic waves, potentially heating the local corona or accelerating the (fast) solar wind (e.g., Velli 1993; Matthaeus et al. 1999; Oughton et al. 2001; Cranmer \& van Ballegooijen 2005; Verdini et al. 2010; van Ballegooijen et al. 2011).

\section{CONCLUSIONS}

We studied combined CoMP and SDO/AIA observations of 14 plumes in the south polar coronal hole on 2011 December 30. Detailed analysis of the characteristic properties of the (propagating) Alfvénic motions and quasi-periodic intensity perturbations highlight the physical richness of the processes taking place in polar plumes, at the base of the fast solar wind. The (perpendicular) Alfvénic waves have an average velocity amplitude of $0.5 \mathrm{~km} \mathrm{~s}^{-1}$, projected phase speed (in the plane of the sky) of $830 \mathrm{~km} \mathrm{~s}^{-1}$, and periods of about 5 minutes. The (parallel) intensity perturbations observed along the same structures have an apparent (projected) speed of $120 \mathrm{~km} \mathrm{~s}^{-1}$ (in both the 171 and $193 \AA$ passbands) and a much longer periodicity of about 15 minutes.

These observations potentially illustrate a scenario where the Alfvénic motions are propagating along, and through, an (longitudinally) inhomogeneous density structure (the polar plume) such that the combination could be a natural progenitor of the MHD turbulence required to accelerate the fast solar wind. However, further direct evidence for the existence of such a turbulent cascade within the plume structures is still needed and might be possible with higher resolution and cadence observations from, for example, the Interface Region Imaging Spectrograph (IRIS). Theoretical modeling is required to investigate whether the small-amplitude intensity perturbations $(\sim 1 \%)$ are sufficiently effective in reflecting the Alfvénic waves to indeed establish a turbulent cascade.

J.L. was a student visitor at HAO. J.L. acknowledges the financial support for his visit to HAO from the Chinese Scholarship Council (CSC). S.W.M. appreciates the support of the Royal Society of Edinburgh and hospitality of the staff in the University of St. Andrews School of Mathematics and Statistics during his extended visit in the summer of 2014. NCAR is sponsored by the National Science Foundation. CoMP data can be found at the MLSO Website. We acknowledge support from NSFC 41131065, 41121003, 973 Key Project 2011CB811403, and CAS Key Research Program KZZD-EW-01-4. We also acknowledge support from NASA contracts NNX08BA99G, NNX11AN98G, NNM12AB40P, NNG09FA40C (IRIS), and NNM07AA01C (Hinode). The research leading to these results has also received funding from the European Commission Seventh Framework Programme (FP7/ 2007-2013) under the grant agreement SOLSPANET (project No. 269299, www.solspanet.eu/solspanet).

\section{REFERENCES}

Banerjee, D., Gupta, G. R., \& Teriaca, L. 2011, SSRv, 158, 267 Banerjee, D., Teriaca, L., Gupta, G. R., et al. 2009, A\&A, 499, L29 Cranmer, S. R., \& van Ballegooijen, A. A. 2005, ApJS, 156, 265 De Moortel, I. 2009, SSRv, 149, 65

De Moortel, I., Antolin, P., \& van Doorsselaere, T. 2015, SoPh, 290, 399 De Moortel, I., Ireland, J., Walsh, R. W., \& Hood, A. W. 2002, SoPh, 209, 61 De Moortel, I., McIntosh, S. W., Threlfall, J., Bethge, C., \& Liu, J. 2014, ApJL, 782, L34

De Moortel, I., \& Nakariakov, V. M. 2012, RSPTA, 370, 3193

De Moortel, I., \& Pascoe, D. J. 2012, ApJ, 746, 31

De Pontieu, B., McIntosh, S. W., Carlsson, M., et al. 2007, Sci, 318, 1574

De Pontieu, B., McIntosh, S. W., Hansteen, V. H., \& Schrijver, C. J. 2009, ApJL, 701, L1

DeForest, C., \& Gurman, J. 1998, ApJL, 501, L217

Gabriel, A. H., Bely-Dubau, F., \& Lemaire, P. 2003, ApJ, 589, 623

Goossens, M., van Doorsselaere, T., Soler, R., \& Verth, G. 2013, ApJ, 768, 191

Lemen, J. R., Title, A. M., Akin, D. J., et al. 2012, SoPh, 275, 17

Lites, B. W., Card, G., Elmore, D. F., et al. 1999, SoPh, 190, 185 
Liu, J., McIntosh, S. W., de Moortel, I., Threlfall, J., \& Bethge, C. 2014, ApJ, 797,7

Liu, J., Zhou, Z., Wang, Y., et al. 2012, ApJL, 758, L26

Matthaeus, W., Zank, G., Oughton, S., Mullan, D., \& Dmitruk, P. 1999, ApJL, 523, L93

McIntosh, S. W., \& De Pontieu, B. 2009, ApJL, 706, L80

McIntosh, S. W., \& De Pontieu, B. 2012, ApJ, 761, 138

McIntosh, S. W., Innes, D. E., De Pontieu, B., \& Leamon, R. J. 2010, A\&A, 510, L2

Nakariakov, V. M. 2006, RSPTA, 364, 473

Newkirk, G., Jr., \& Harvey, J. 1968, SoPh, 3, 321

Ofman, L., Nakariakov, V. M., \& DeForest, C. E. 1999, ApJ, 514, 441

Oughton, S., Matthaeus, W., Dmitruk, P., et al. 2001, ApJ, 551, 565

Parker, E. N. 1991, ApJ, 372, 719

Pascoe, D. J., Hood, A. W., de Moortel, I., \& Wright, A. N. 2013, A\&A, 551, A40

Pascoe, D. J., Wright, A. N., \& de Moortel, I. 2010, ApJ, 711, 990
Threlfall, J., De Moortel, I., McIntosh, S. W., \& Bethge, C. 2013, A\&A, 556, A124

Tian, H., McIntosh, S. W., \& de Pontieu, B. 2011, ApJL, 727, L37

Tomczyk, S., Card, G., Darnell, T., et al. 2008, SoPh, 247, 411

Tomczyk, S., \& McIntosh, S. W. 2009, ApJ, 697, 1384

Tomczyk, S., McIntosh, S. W., Keil, S. L., et al. 2007, Sci, 317, 1192

Torrence, C., \& Compo, G. P. 1998, BAMS, 79, 61

van Ballegooijen, A., Asgari-Targhi, M., Cranmer, S., \& DeLuca, E. 2011, ApJ, 736, 3

Velli, M. 1993, A\&A, 270, 304

Verdini, A., Velli, M., Matthaeus, W. H., Oughton, S., \& Dmitruk, P. 2010, ApJL, 708, L116

Wang, H. 1998, ApJ, 509, 461

Wilhelm, K. 2000, A\&A, 360, 351

Withbroe, G. L., \& Noyes, R. W. 1977, ARA\&A, 15, 363

Yamauchi, Y., Suess, S. T., \& Sakurai, T. 2003, in AIP Conf. Ser. 679, Solar Wind Ten, ed. M. Velli et al. (Melville, NY: AIP), 255 